\title{
Valoración emocional de imágenes y temperamento en preescolares: papel de la edad y la valencia
}

\author{
Verónica Adriana Ramírez ab*, María Soledad Segretin a , Sebastián Lipina a \\ y Eliana Ruetti ab
}

\author{
a Unidad de Neurobiología Aplicada, UNA, CEMIC-CONICET, Buenos Aires, Argentina \\ ${ }^{b}$ Facultad de Psicología, Universidad de Buenos Aires, Buenos Aires, Argentina
}

Recibido el 11 de noviembre de 2019; aceptado el 13 de julio de 2020

\author{
PALABRAS CLAVE \\ Valoración emocional, \\ temperamento, \\ desarrollo emocional, \\ preescolares, \\ diferencias individuales
}

\begin{abstract}
Resumen La valoración es una etapa crucial del procesamiento emocional que prepara para la acción. Durante este proceso se generan distintas respuestas a partir de la evaluación de aspectos emocionales de los estímulos. Estas variaciones pueden deberse a la influencia de características individuales. La literatura señala al temperamento como uno de los factores asociados a las diferencias en la valoración emocional y el afrontamiento. Este trabajo analiza la relación entre la valoración emocional de estímulos visuales y características temperamentales obtenidas por medio del Cuestionario de Conducta Infantil (CBQ). Para esto, 198 preescolares de cuatro y cinco años valoraron 15 imágenes (negativas, neutras y positivas) y se analizaron estas valoraciones en función de las características temperamentales. Se encontró mayor cantidad de valoraciones negativas a los cuatro años que a los cinco $\left(p=.056, \eta^{2}\right.$ parcial $\left.=.031\right)$, y de valoraciones positivas en el grupo con puntaje alto de esfuerzo de control en comparación con el de puntaje bajo $\left(p=.020, n^{2}\right.$ parcial $\left.=.029\right)$. Esto sugiere una asociación entre la valoración emocional, la edad y el esfuerzo de control. Este resultado podría deberse a que las niñas y los niños estuvieran desviando su atención de los aspectos negativos de los estímulos.
\end{abstract}

(c) 2020 Fundación Universitaria Konrad Lorenz. Este es un artículo Open Access bajo la licencia CC BY-NC-ND (http://creativecommons.org/licenses/by-nc-nd/4.0/).

Emotional appraisal of images and temperament in preschoolers: Role of age and valence

Abstract Emotional appraisal is a crucial stage of emotional processing that prepares for action (coping). During this process different responses are generated from the evaluation of emotional aspects of the stimuli. These variations may be due to the influence of individual characteristics. The literature points to temperament as one of the factors associated with differences in emotional appraisal and coping. This paper analyzes the relationship between the emotional appraisal of visual stimuli and temperamental characteristics, obtained through the Children's Behavior Questionnaire (CBQ). For this purpose, 198 preschoolers aged 4 and 5 assigned

\footnotetext{
* Autor para correspondencia.
}

Correo electrónico: veronica.adriana.ramirez@gmail.com

https://doi.org/10.14349/sumapsi.2020.v27.n2.2

ISSN 0121-4381, ISSN-E 2145-9797/@ 2020 Fundación Universitaria Konrad Lorenz. Este es un artículo Open Access bajo la licencia CC BY-NC-ND (http://creativecommons.org/licenses/by-nc-nd/4.0/). 
one of three possible emotional expressions to 15 images (negative, neutral and positive) and then compared the number of appraisals according to temperamental characteristics. Higher number of negative appraisals were found in the 4-year group compared to the 5-year group $\left(p=.056, \eta^{2}\right.$ partial $\left.=.031\right)$, and of positive appraisals in the group with high effortful control score compared to the low score group $\left(p=.020, \eta^{2}\right.$ partial $\left.=.029\right)$, suggesting an association between emotional assessment, age and effortful control. This result could be due to the fact that children were diverting they attention from the negative aspects of stimuli.

(c) 2020 Fundación Universitaria Konrad Lorenz. This is an open access article under the CC BYNC-ND license (http://creativecommons.org/licenses/by-nc-nd/4.0/).

Existe acuerdo entre numerosas investigaciones acerca de cómo las emociones comprenden diferentes procesos, entre los cuales se encuentran los procesos de valoración, la tendencia a la acción o afrontamiento, el comportamiento, diversos cambios fisiológicos y expresiones subjetivas (Scherer \& Fontaine, 2018). Uno de los componentes básicos del temperamento lo constituyen las diferencias individuales en el procesamiento y la regulación emocional (Andrés et al., 2017; Rothbart, Bates, Damon \& Lerner, 2006; Rothbart, Sheese \& Posner, 2007).

Específicamente, la valoración emocional puede definirse como el proceso a través del cual se generan diferentes respuestas ante un estímulo a partir de evaluaciones subjetivas (Fernando, Kashima \& Laham, 2015; Kuppens, Champagne \& Tuerlinckx, 2012; Parsafar \& Davis, 2018; Scherer \& Fontaine, 2018). La valoración emocional se refiere a la información que confiere un significado personal a los eventos percibidos, la cual se extrae de los estímulos ambientales y resulta de la interacción entre las propiedades de estos estímulos y de la sensibilidad individual ante ellos (Ridderinkhof, 2017; Yih, Uusberg, Taxer \& Gross, 2018).

Las personas realizan constantemente valoraciones emocionales acerca de los estímulos de acuerdo con lo que implican para su propio bienestar, sus objetivos e inquietudes (Kuppens et al., 2012). Sin embargo, estas valoraciones y las consecuentes reacciones que se desencadenan suelen variar entre las personas, en especial entre las niñas y los niños, incluso cuando se dan ante un mismo estímulo (Fernando et al., 2015; Roseman \& Smith, 2001). Por una parte, esto sugiere que, en el propósito de definir una emoción y sus respuestas asociadas, resulta crucial analizar la valoración tanto como las propiedades del estímulo en sí mismo (Fernando et al., 2015). Por otra parte, esto implica, además, que esas valoraciones podrían modular las características individuales (e.g. edad, sexo, temperamento) (Fivush \& Baker-Ward, 2005; Wang, 2008).

Existe evidencia sobre cómo el temperamento influencia el modo en que se valoran las situaciones y las estrategias de afrontamiento desplegadas (Lengua \& Long, 2002; Rothbart, 2011). El temperamento puede definirse como el conjunto de diferencias individuales relativamente estables en la reactividad emocional y la autorregulación, biológicamente basadas e influenciadas a lo largo del tiempo por la maduración y la experiencia (Bornstein, Hahn, Putnick \& Pearson, 2018; Posner \& Rothbart, 2018; Rothbart, Ahadi, Hersey \& Fisher, 2001; Rueda \& Rothbart, 2009; Thompson, Zalewski \& Lengua, 2014). La reactividad refiere a la activación de los sistemas de respuesta motora, afectiva y sensorial. La autorregulación, por su parte, se refiere a procesos que sirven para modificar (aumentar o disminuir) la reactividad, incluyendola atención yel control inhibitorio. Las diferentes características temperamentales afectarían la codificación e interpretación de señales internas y externas, lo que daría como resultado variaciones en las valoraciones que llevarían a la generación de distintas emociones y culminarían en diversos patrones de acción (Derryberry \& Rothbart, 1997; Lemerise \& Arsenio, 2000; Lengua \& Long, 2002; Scherer \& Moors, 2017).

La literatura da cuenta de diferentes asociaciones entre temperamento y procesos emocionales en diferentes edades. Por ejemplo, desde la perspectiva conceptual de Rothbart y sus colaboradores se ha encontrado que puntajes altos en la escala de esfuerzo de control se relacionaron con puntajes más altos en variables relacionadas con empatía, sensibilidad, logros académicos y regulación emocional en situaciones sociales en general (adultos: Bridgett, Oddi, Laake, Murdock \& Bachmann, 2013; niñas y niños de entre siete meses y siete años: Posner, Rothbart, Sheese \& Voeker, 2014). Asimismo, se ha encontrado que puntajes altos en la dimensión afectividad negativa se relacionaron con mayores signos de angustia durante el período escolar, así como con dificultades para regular la atención frente a estímulos emocionales, especialmente aquellos relacionados con amenazas (infantes de entre tres meses y tres años: Conejero \& Rueda, 2018; Gartstein, Putnam \& Rothbart, 2012).

Por otra parte, se ha encontrado que altos puntajes en extraversión se relacionaron con un aumento en la tendencia al compromiso, la impulsividad y una alta actividad social (infantes de entre tres y 18 meses: Fields-Olivieri, Cole \& Maggi, 2017; Gartstein et al., 2012). Por último, también se verificaron asociaciones entre estas dimensiones temperamentales, como, por ejemplo, una relación inversa entre la dimensión afectividad negativa y las de esfuerzo de control y extraversión (adultos: Evans \& Rothbart, 2009; niñas y niños de tres y cuatro años: Rothbart et al., 2011). Estos estudios permitieron evidenciar asociaciones específicas entre determinados aspectos del temperamento y de componentes emocionales. A pesar de que los resultados no son concluyentes, existe consenso acerca de cómo las conductas relacionadas con las emociones tienen algún tipo de relación con los perfiles de temperamento (MacNeill \& Pérez-Edgar, 2020). Sin embargo, pocos estudios incluyen poblaciones de edad preescolar.

En particular, se encuentran unos pocos estudios que analizan la relación entre rasgos temperamentales y la valoración emocional. Por ejemplo, en un estudio realizado en niñas y niños de ocho a 12 años se encontró que, ante un evento negativo, niveles altos de afectividad negativa provocaban valoraciones más negativas de los estímulos, lo que llevaba a un mayor uso de las capacidades de afrontamien- 
to, mientras que la extraversión facilitaba visiones optimistas de este, de modo que se valoraba como desafiante e incrementaba así la confianza en los propios recursos de afrontamiento (Lengua \& Long, 2002; Lengua, Sandler, West, \& Wolchik, 1999). Es decir, altos niveles de afectividad negativa y extraversión se asociaban a diferentes valoraciones, lo cual generaba un mayor ajuste en las capacidades de afrontamiento de las niñas y los niños.

En nuestro conocimiento, la relación entre las características de estas dimensiones temperamentales y la valoración emocional durante etapas tempranas del desarrollo no ha sido estudiada en profundidad. De acuerdo con lo planteado, el objetivo de este trabajo es analizar el desempeño de preescolares de cuatro y cinco años, en una tarea de valoración de imágenes con diferente contenido emocional (negativo, neutro y positivo) y sus características temperamentales por medio del reporte familiar.

\section{Método}

\section{Participantes}

Se conformó una muestra incidental de 198 niñas y niños de cuatro y cinco años, contrabalanceada según género (99 niñas) (véase la tabla 1), que concurrían a cuatro jardines de infantes de los barrios de Lugano, Mataderos y Almagro de la Ciudad Autónoma de Buenos Aires (dos jardines de gestión pública: $81 \%$ de las y los participantes, y dos de gestión privada: $19 \%$ de las y los participantes). La información socioambiental de los hogares se obtuvo a partir de entrevistas con familiares, las cuales, en muchos casos, no fue posible realizar por no haberse presentado ningún familiar o por tener que retirarse antes de completar la totalidad de los cuestionarios. Por este motivo, solo se cuenta con información de 121 familias. Ninguna y ninguno de las niñas y los niños presentaba trastornos del desarrollo en términos de su historial de salud peri y postnatal, según lo reportado por las familias, las directivas de los establecimientos educativos y la consulta de legajos. Todas y todos tenían como lengua materna el español y eran de nacionalidad argentina o vinieron al país dentro de los primeros dos años de vida. La edad promedio materna era de 33.11 años $(D E=7.56)$ y paterna de 35.13 años $(D E=8.09)$. El nivel educativo de las familias se distribuía de la siguiente forma: el 5.35\% de los padres y el $4.13 \%$ de las madres no tenían estudios o tenían primario incompleto, el $44.65 \%$ de los padres y el $43.8 \%$ de las madres tenían primario completo o secundario incompleto, el $29.46 \%$ de los padres y el $26.45 \%$ de las madres tenían secundario completo o terciario incompleto, mientras que el $20.54 \%$ de los padres y el $25.62 \%$ de las madres tenían por lo menos terciario completo. Como criterios de inclusión se consideró que las y los participantes tuvieran cuatro o cinco años, no tuvieran antecedentes de salud y el español fuera su primera lengua.

\section{Instrumentos}

Se implementó un diseño exploratorio sincrónico por medio del cual se administró una tarea de valoración de imágenes con diferentes estímulos y una entrevista a los y las responsables de las niñas y los niños a fin de obtener el reporte de su temperamento.
Tabla 1 Composición de la muestra en función de edad y sexo

\begin{tabular}{|c|c|c|c|c|c|c|}
\hline \multirow{3}{*}{ Edad } & \multicolumn{6}{|c|}{ Sexo } \\
\hline & \multicolumn{2}{|c|}{ Femenino } & \multicolumn{2}{|c|}{ Masculino } & \multicolumn{2}{|c|}{ Total } \\
\hline & $n$ & $\%$ & $n$ & $\%$ & $n$ & $\%$ \\
\hline Cuatro años & 78 & 39.39 & 72 & 36.36 & 150 & 75.75 \\
\hline Cinco años & 21 & 10.61 & 27 & 13.64 & 48 & 24.25 \\
\hline Total & 99 & 50 & 99 & 50 & 198 & 100 \\
\hline
\end{tabular}

\section{Tarea de valoración emocional}

La tarea consistía en observar y valorar una serie de 15 imágenes obtenidas de la Developmental Affective Photo System (DAPS, Cordon, Melinder, Goodman \& Edelstein, 2013), seleccionadas en función de estudios previos realizados por los autores de este trabajo (Ruetti, Segretin, Ramírez \& Lipina, 2019). Todas las imágenes estaban en color, tenían personas (e.g., niñas o niños, mujeres), animales (e.g., perros, cucarachas), objetos (e.g., un libro, una taza), o personas realizando acciones (e.g., niños jugando con una pelota, niña saltando la soga). El criterio para la selección de las imágenes fue que estuvieran compuestas por los mismos elementos (e.g. misma cantidad de personas, acciones), que no fuesen ambiguas en sus valencias y fuera posible especificarlas con una palabra o frase. Estas imágenes se presentaban mediante una netbook o tablet. La serie la conformaban cinco imágenes negativas, cinco neutras y cinco positivas, dispuestas de forma tal que no quedaran más de dos imágenes consecutivas de la misma valencia. La secuencia de imágenes fue la misma en todos los casos. Antes de dicha serie se les mostraban dos imágenes, una en blanco para captar la atención de las niñas y los niños en la presentación de la consigna, y otra de práctica con el fin de corroborar su comprensión. La valoración emocional se realizaba por medio de un juego de caras con expresiones emocionales (una negativa, una neutra y una positiva), solicitando la asignación para cada imagen de una cara de las tres posibles. El intervalo entre la presentación de cada imagen variaba entre participantes, ya que cada cual decidía cuándo pasar a la observación de la siguiente imagen. Sin embargo, en promedio, este intervalo no fue mayor a tres segundos. Se consideró como variable de interés la cantidad de valoraciones de cada una de las valencias (positiva, neutra, negativa) ante la visualización de las imágenes. Teniendo en cuenta que cada niña y niño valoró un total de 15 imágenes, cada variable dependiente pudo adoptar valores entre 0 y 15 , de modo que sumaron entre las tres un total de 15 valoraciones.

\section{Cuestionario de Conducta Infantil (CBQ, por sus siglas en inglés, Rothbart et al., 2001)}

Por medio de este cuestionario es posible indagar dimensiones del temperamento mediante el reporte de responsables de las niñas y los niños. Estas dimensiones incluyen aspectos de reactividad emocional negativa (miedo, estrés ante las limitaciones, tristeza), extraversión (risas, sonrisas, placer) y mecanismos de autorregulación de la atención, la actividad, la inhibición conductual (miedo o timidez) y el control inhibitorio. Estas dimensiones 
presentaron adecuada consistencia interna, con rangos de .72 a .75 , estabilidad en el tiempo, así como un buen ajuste a la estructura de tres factores (Putnam \& Rothbart, 2006). Las propiedades psicométricas de la versión en español fueron reportadas por De la Osa, Granero, Penelo, Domènech y Ezpeleta (2014), quienes realizaron un análisis factorial y encontraron una estructura factorial aceptable (KMO = .76) con tres dimensiones que explicaron el $22.6 \%$ de la varianza. Además, reportaron índices de consistencia interna apropiados: extraversión ( $\alpha=.65$ ), afectividad negativa $(\alpha=.77$ ) y esfuerzo de control $(\alpha=.66)$. Se administró la versión breve en español del cuestionario de Putnam y Rothbart (2006) para niñas y niños de tres a siete años, realizada por el Grupo de Investigación en Psicología Evolutiva (GIPSE) de la Universidad de Murcia. Las 36 afirmaciones conforman tres dimensiones del temperamento, extraversión, afectividad negativa y esfuerzo de control. Cada dimensión se compone de 12 afirmaciones sobre la conducta de niñas y niños, acerca de las cuales cada madre o padre responsable debe establecer el grado de acuerdo o desacuerdo con estas en una escala tipo Likert de siete valores. De este modo, cada afirmación puede tomar un puntaje de 0 a 7 y el puntaje de cada dimensión se halla al promediar los puntajes de las afirmaciones que la componen, por lo que también toma valores de 0 a 7 . En el presente estudio se calcularon las medianas para cada dimensión (extraversión: $M d=4.42$; afectividad negativa: $M d=4.42$; esfuerzo de control: $M d=5.46$ ) y se dividió a la muestra en función de estos valores. De esta manera, cada dimensión quedó dividida en un grupo de valores bajos, correspondientes a aquellos puntajes que coinciden con la mediana o están por debajo de ella, y otro de valores altos con puntajes por encima de la mediana. En la tabla 2 figuran los valores máximos y mínimos, los tamaños de la muestra, las medias y los desvíos típicos de los puntajes para cada grupo de cada dimensión.

\section{Procedimiento}

Se aplicaron los procedimientos recomendados por la American Psychological Association (1992) y por el Ethical Research Involving Children Project (ERIC) (Graham, Powell, Taylor, Anderson \& Fitzgerald, 2013), además de los principios establecidos por la Convención Internacional sobre los Derechos del Niño y la Ley Nacional N²6.061 de Protección Integral de los Derechos del Niño. Una vez obtenida la autorización del Ministerio de Educación del Gobierno de la
Ciudad de Buenos Aires y de las instituciones escolares en las que se propuso realizar las actividades, se organizaron reuniones informativas con las familias que tuvieron oportunidad de interactuar con los miembros del grupo de investigación. Consecuentemente, se invitó a estas familias a participar y a autorizar la participación de sus hijas e hijos, para lo cual fue condición necesaria la firma de consentimientos informados. Los procedimientos implementados no presentaron ningún riesgo y fueron aprobados por el Comité de Ética del CEMIC (Protocolo $N^{\circ}$ 961).

La tarea aquí presentada forma parte de un estudio más amplio en el que se evaluaron otros procesos cognitivos en sesiones distintas. Todas las evaluaciones y las entrevistas se realizaron de forma individual por un operador o una operadora. La sesión con las niñas y los niños tuvo una duración de diez minutos, aproximadamente, mientras que las entrevistas con las y los responsables insumieron alrededor de 30 minutos, tiempo durante el cual se les administraba el CBQ.

Antes de cada sesión se pedía autorización a las docentes para retirar a los niños y las niñas de sus salas con un operador o una operadora. Una vez en el salón de evaluación, cada cual se sentaba en una mesa diferente frente a un operador o una operadora. Previo a comenzar la evaluación se mostraban los materiales: tres caras con diferentes expresiones emocionales (una cara feliz, una cara triste y una neutra) y la netbook o tablet en la que se presentarían las imágenes. En primer lugar, aparecía una pantalla en blanco con el fin de focalizar la atención en el dispositivo, mientras se brindaba la consigna: "Hoy vamos a ver unas fotos en la pantalla y vos me tenés que decir cómo te sentís cuando ves esa foto. Para eso, vamos a usar estas caritas". A continuación, se señalaba una a una cada carita y se preguntaba cómo estaba esa carita, con la finalidad de verificar la comprensión de la expresión emocional de esta. Luego, se pasaba a la siguiente imagen, una lamparita, con el propósito de practicar y comprobar la comprensión de la consigna: "Vamos a practicar. Mirá la foto y señala una carita para decirme cómo te sentiste cuando viste la foto". Después de realizada la valoración de la imagen de práctica, se preguntaba si se había sentido como la carita señalada (“¿Así te sentiste?, ¿feliz?”). En caso de afirmar se continuaba con la prueba, pero si negaba sentirse de esa forma se repetía la consigna reforzando la intención de que no había respuestas correctas, que solo queríamos saber cómo se sentía al ver las imágenes. Cada operador y operadora consignaba la respuesta en una planilla de forma

Tabla 2 Descripción de los puntajes de temperamento para cada dimensión y grupo

\begin{tabular}{|c|c|c|c|c|c|}
\hline Dimensiones de temperamento & $N$ & Mínimo & Máximo & $M$ & $D E$ \\
\hline Baja extraversión & 106 & 2.25 & 4.42 & 3.79 & 0.51 \\
\hline Alta extraversión & 92 & 4.43 & 6.17 & 4.95 & 0.35 \\
\hline Baja afectividad negativa & 100 & 2.67 & 4.42 & 3.97 & 0.38 \\
\hline Alta afectividad negativa & 98 & 4.45 & 6.25 & 5.02 & 0.46 \\
\hline Bajo esfuerzo de control & 100 & 3.33 & 5.46 & 4.85 & 0.49 \\
\hline Alto esfuerzo de control & 98 & 5.50 & 7.00 & 6.02 & 0.37 \\
\hline
\end{tabular}

Nota. $N$ = tamaño de muestra, $M=$ media, $D E$ = desvío estándar. 
simultánea a la realización de la tarea. Se excluyeron de la muestra aquellos casos en los que se observaron todas las valoraciones de una misma valencia por considerarse una falta de discriminación entre los diferentes valores de la variable o bien de motivación o interés para la realización de la tarea.

\section{Análisis de datos}

En primer término, se efectuaron análisis univariados de las variables dependientes que incluyeron la determinación de tamaños muestrales, medias y desvíos estándar para cada grupo. Luego se evaluó el cumplimiento de los criterios de normalidad y homocedasticidad. A fin de analizar las comparaciones de la valoración de las imágenes entre los grupos, se realizó un análisis multivariado de la varianza (Manova), para lo cual se tomó como variables dependientes la cantidad de valoraciones (negativa, neutra y positiva), y como independientes el tipo temperamental de cada dimensión (alto o bajo para extroversión, afectividad negativa y esfuerzo de control), en las y los preescolares de cuatro y cinco años. A fin de llevar a cabo los análisis descriptivos e inferenciales se utilizó el software SPSS 21.

\section{Resultados}

En primera instancia se establecieron las medias y los desvíos estándar para las variables dependientes (véase la tabla 3). En todos los casos se verificó el cumplimiento de los criterios de normalidad y homocedasticidad, por lo que se procedió a utilizar los análisis paramétricos planificados.

El análisis múltiple de varianza intergrupo implementado indicó: (1) la ausencia de diferencias según sexo $(F=0.503$, $p=.606)$, características temperamentales de extraversión $(F=0.732, p=.482)$, afectividad negativa $(F=0.683, p=.506)$ y esfuerzo de control $(F=2.238, p=.109)$, así como de la interacción entre los grupos de características temperamentales; y (2) una asociación de carácter marginal en función de la edad $\left(F=2.928, p=.056, \eta^{2}\right.$ parcial $\left.=.031\right)$, indicando diferencias en la valoración emocional entre preescolares de cuatro y cinco años (véase la tabla 4). Estas diferencias se describen a continuación en los análisis intragrupo.

A continuación, se analizaron las diferencias halladas en el análisis intergrupo. Se encontró que las diferencias por edad en la cantidad de valoraciones solo eran significativas para las de valencia negativa $(F=5.547, p=.020$, $\eta^{2}$ parcial $\left.=.029\right)$, con un tamaño del efecto moderado, pero no para imágenes con valencia neutra $(F=1.262$, $p=.263)$ o positiva $(F=0.699, p=.404)$, encontrándose una mayor cantidad de valoraciones negativas a los cuatro años con respecto a los cinco años (figura 1).

Al comparar las valoraciones según los grupos temperamentales, se identificaron diferencias significativas en la cantidad de valoraciones de acuerdo con el esfuerzo de control $(F=4.467, p=.036)$, con un tamaño del efecto moderado $\left(n^{2}\right.$ parcial $\left.=.031\right)$, siendo mayor la cantidad de valoraciones positivas para el grupo de alto esfuerzo de control (figura 2), pero no así en las de valencia negativa $(F=1.789, p=.183) 0$ neutras $(F=1.650, p=.201)$. Los análisis no arrojaron diferencias significativas para el resto de los factores.
Tabla 3 Descripción de las valoraciones realizadas según los grupos de temperamento

\begin{tabular}{|c|c|c|c|c|}
\hline $\begin{array}{c}\text { Grupo de } \\
\text { temperamento }\end{array}$ & Valoraciones & $N$ & $M$ & $D E$ \\
\hline \multirow[t]{3}{*}{ Baja extraversión } & Negativas & 106 & 4.30 & 1.64 \\
\hline & Neutras & 106 & 4.33 & 1.57 \\
\hline & Positivas & 106 & 6.37 & 2.00 \\
\hline \multirow[t]{3}{*}{ Alta extraversión } & Negativas & 92 & 4.55 & 1.48 \\
\hline & Neutras & 92 & 4.50 & 1.90 \\
\hline & Positivas & 92 & 5.95 & 2.09 \\
\hline \multirow[t]{3}{*}{ Baja afectividad negativa } & Negativas & 100 & 4.34 & 1.67 \\
\hline & Neutras & 100 & 4.32 & 1.76 \\
\hline & Positivas & 100 & 6.34 & 2.25 \\
\hline \multirow[t]{3}{*}{ Alta afectividad negativa } & Negativas & 98 & 4.50 & 1.47 \\
\hline & Neutras & 98 & 4.50 & 1.70 \\
\hline & Positivas & 98 & 6.00 & 1.82 \\
\hline \multirow[t]{3}{*}{ Bajo esfuerzo de control } & Negativas & 100 & 4.60 & 1.62 \\
\hline & Neutras & 100 & 4.52 & 1.59 \\
\hline & Positivas & 100 & 5.88 & 1.86 \\
\hline \multirow[t]{3}{*}{ Alto esfuerzo de control } & Negativas & 98 & 4.23 & 1.50 \\
\hline & Neutras & 98 & 4.30 & 1.87 \\
\hline & Positivas & 98 & 6.47 & 2.20 \\
\hline
\end{tabular}

Nota. $N$ = tamaño de muestra, $M=$ media, $D E$ = desvío estándar.

Tabla 4 Comparaciones entre los diferentes grupos para identificar la presencia de diferencias en la valoración de imágenes en función de las dimensiones del temperamento de las niñas y los niños

\begin{tabular}{|c|c|c|c|}
\hline Variables & $F$ & $p$ & $\eta^{2}$ parcial \\
\hline Edad & 2.928 & .056 & .031 \\
\hline Sexo & 0.503 & .606 & .005 \\
\hline Extraversión & 0.732 & .482 & .008 \\
\hline Afectividad negativa & 0.683 & .506 & .007 \\
\hline Esfuerzo de control & 2.238 & .109 & .024 \\
\hline Extraversión $\mathrm{x}$ afectividad negativa & 0.456 & .634 & .005 \\
\hline Extraversión $x$ esfuerzo de control & 1.124 & .327 & .012 \\
\hline $\begin{array}{l}\text { Afectividad negativa } x \text { esfuerzo } \\
\text { de control }\end{array}$ & 0.423 & .655 & .005 \\
\hline $\begin{array}{l}\text { Extraversión } x \text { afectividad negativa } \\
x \text { esfuerzo de control }\end{array}$ & 0.125 & .883 & .001 \\
\hline
\end{tabular}

Nota. $N$ = tamaño muestral, $\eta^{2}$ parcial = tamaño del efecto. $\mathrm{El}$ valor de $p$ fue fijado en .05 . 


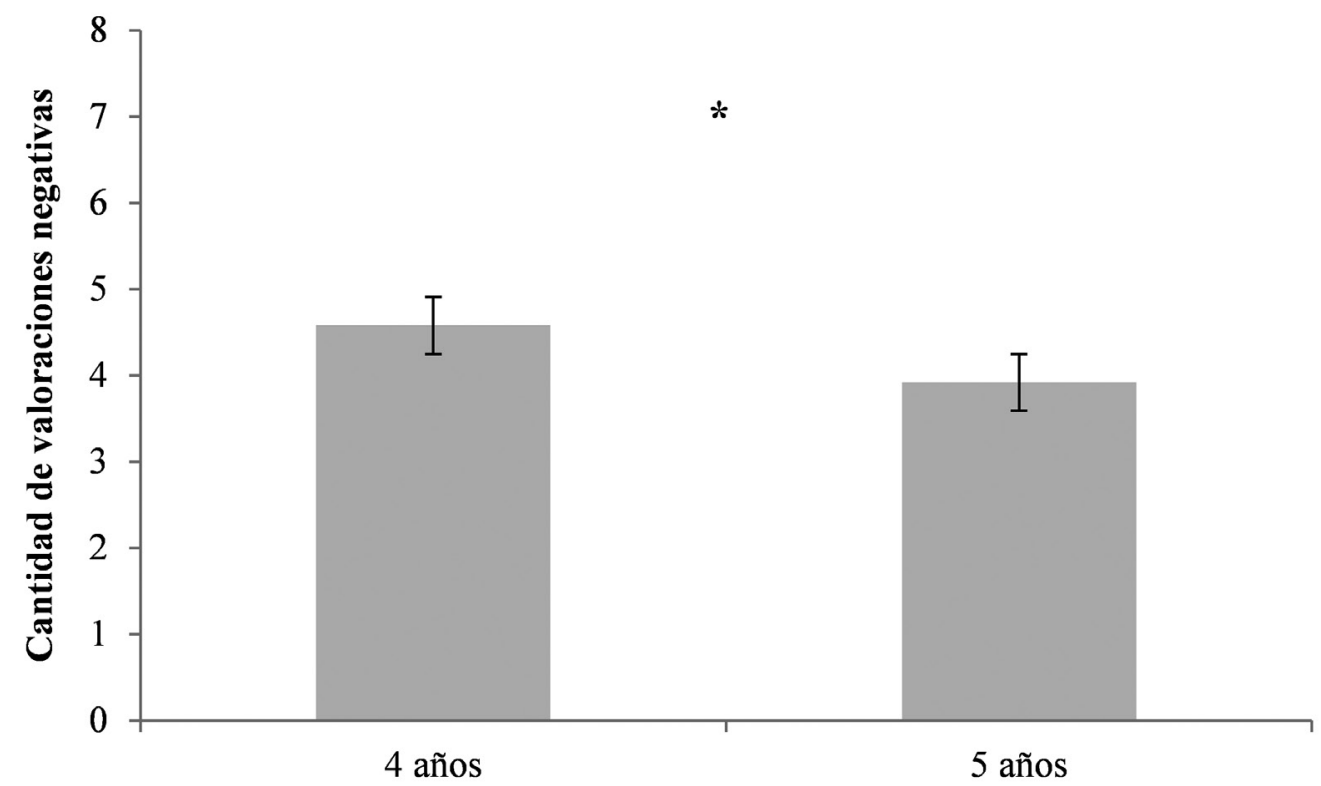

Figura 1. Promedio de cantidad de valoraciones negativas por edad.

\section{Discusión}

En este trabajo se realizó un estudio orientado a analizar el papel de las características temperamentales de preescolares de cuatro y cinco años en una tarea de valoración de imágenes con diferente contenido emocional. Se encontraron diferencias en las valoraciones negativas, pero no en neutras ni positivas, así como tampoco en las comparaciones de acuerdo con el sexo, ni en las características temperamentales de extraversión y afectividad negativa. Las variaciones en la valoración emocional en función de la edad han sido reportadas en trabajos previos, al igual que la falta de asociación del sexo con las respuestas de valoración (Jaume, Ruetti, Segretin \& Lipina, 2016). Este perfil de resultados difiere con respecto a las relaciones con aspectos de procesamiento emocional, como, por ejemplo, la regulación emocional ante rostros amenazantes en niñas y niños más pequeños (Conejero \& Rueda, 2018) y la extraversión (Reyna \& Brussino, 2015). Lo anterior sugiere que en diferentes etapas del desarrollo tales relaciones podrían expresar cambios específicos que deben analizarse en futuras investigaciones.

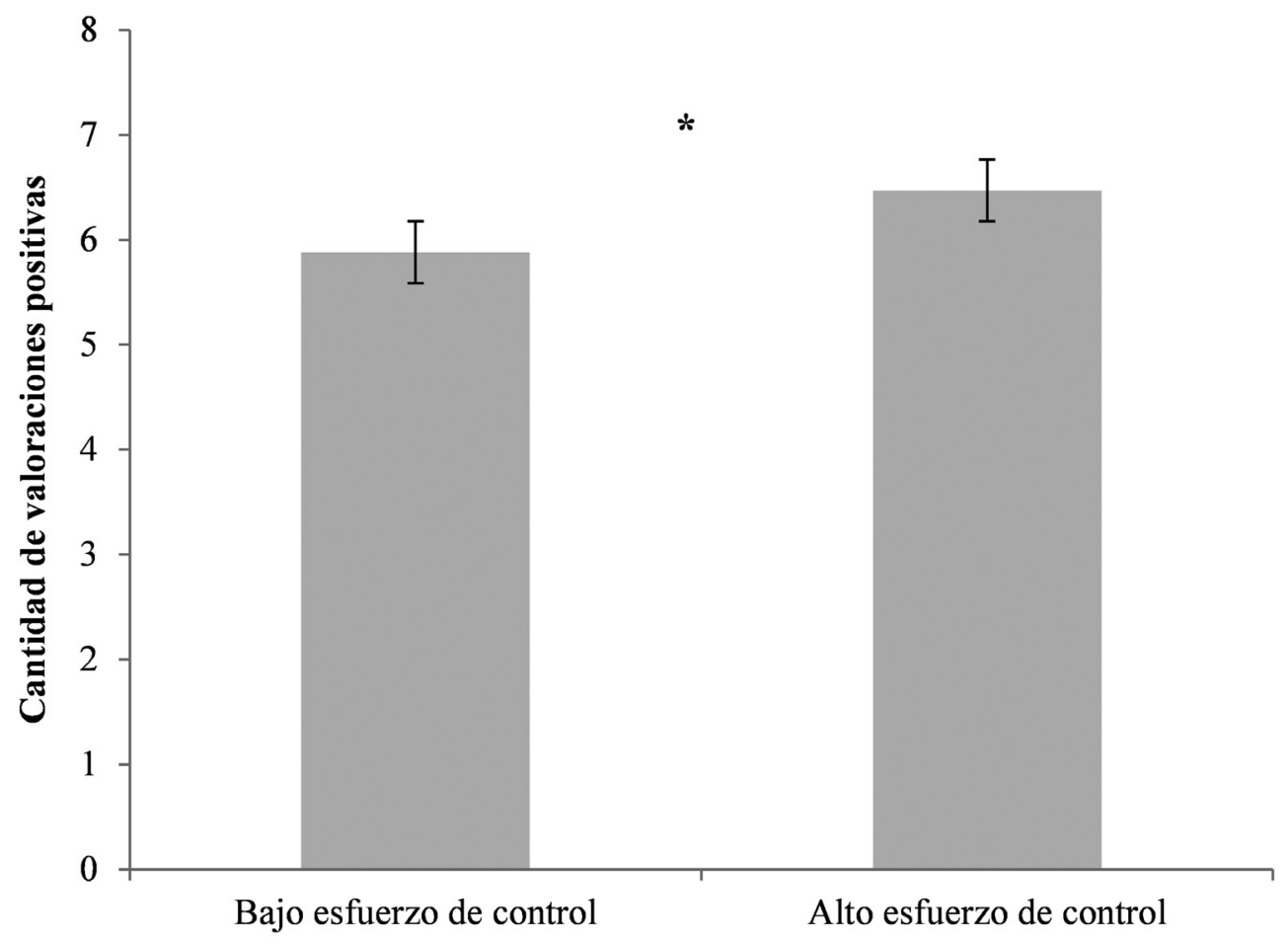

Figura 2. Promedio de cantidad de valoraciones positivas por grupo de esfuerzo de control. 
Por otra parte, son escasos los antecedentes en la literatura que refieran al rol de las características temperamentales de niñas y niños en la valoración emocional. En específico, Lengua y sus colaboradores llevaron a cabo estudios con niñas y niños de ocho a 12 años, relacionando patrones de afrontamiento, rasgos temperamentales y valoración emocional de eventos estresantes (Lengua \& Long, 2002; Lengua et al., 1999). Los resultados de estos estudios indican que quienes tenían mayores rasgos temperamentales de emocionalidad negativa valoraron los eventos como más amenazantes con relación a aquellos con mayor cantidad de rasgos de emocionalidad positiva. En el presente trabajo se verificaron diferencias en la valoración de imágenes positivas entre el grupo con alto y bajo esfuerzo de control, es decir, en otro factor temperamental y en un grupo etario menor. El esfuerzo de control incluye las habilidades para administrar de forma voluntaria la atención e inhibir o activar un comportamiento necesario para procesos de adaptación. Por ejemplo, las habilidades para centrar la atención cuando hay distracciones, no interrumpir a otros, quedarse quietas o quietos en clase y obligarse a realizar una tarea desagradable son aspectos del esfuerzo de control (Rothbart et al., 2006).

Una posible relación entre un puntaje alto en esfuerzo de control y una mayor cantidad de valoraciones positivas podría deberse a que las niñas y los niños estuviesen desviando su atención de aspectos negativos de los estímulos (Conejero \& Rueda, 2018; Thompson et al., 2014). La falta de relación entre las otras medidas temperamentales y la valoración emocional puede deberse a que las categorías emocionales se encuentran en proceso de desarrollo durante la etapa preescolar (Rothbart et al., 2011). Sin embargo, se requerirían estudios adicionales, como, por ejemplo, analizar el papel de las características temperamentales de niñas y niños ante la valoración de tareas con mayor complejidad y en un rango más amplio de edades.

Por otra parte, algunas implicancias que podrían tener las diferencias en la valoración emocional se relacionan con la selección e implementación de distintas estrategias de afrontamiento. Como se mencionó, las valoraciones emocionales generan diferentes tendencias a la acción o estrategias de afrontamiento ante el estímulo valorado (Lengua \& Long, 2002; Lengua et al., 1999; Rothbart, 2011; Thompson et al., 2014). Durante el proceso de valoración emocional se identifican relaciones entre el individuo y el estímulo que, en razón a la necesidad de establecerse o modificarse, dan lugar a la acción (Ridderinkhof, 2017). Estas estrategias de afrontamiento pueden definirse como esfuerzos dinámicos, tanto cognitivos como conductuales, dirigidos a manejar demandas externas o internas valoradas de acuerdo con los recursos de la persona, los cuales dan como resultado la adaptación (Holodynski \& Seeger, 2019; Lengua \& Long, 2002; Rothbart, 2011). De este modo, se plantea que las estrategias de afrontamiento se encuentran influenciadas por la valoración emocional, ya que dependiendo de cómo sea la valoración del estímulo será la respuesta que se presente ante él. Una forma de profundizar estos hallazgos sería a través de un estudio en el que la valoración emocional de preescolares permitiese desplegar distintas estrategias de afrontamiento en un mismo diseño.

Existen algunas limitaciones en este trabajo que deben discutirse para interpretar de forma adecuada los resulta- dos. Por una parte, la utilización de un cuestionario como medida de las características temperamentales de niñas y niños implica la utilización de un reporte familiar para la obtención en el abordaje del temperamento infantil, de modo que el empleo de otras medidas de observación de las características temperamentales complementaría este abordaje (Stifter \& Dollar, 2016). Por otra, las respuestas otorgadas por las familias podrían verse influenciadas por factores individuales y socioambientales que no han sido analizados en este estudio. Los factores que podrían modular la percepción familiar acerca del temperamento infantil son la cantidad de horas al cuidado de las niñas y los niños, edad materna o paterna, nivel de educación, ocupación, nivel de ingresos económicos y las condiciones de vida del hogar, cuyas variaciones no se analizaron aquí por no contarse con la totalidad de dicha información de las familias participantes. Contar con información proveniente de otras fuentes (e.g. otros familiares, cuidadores o docentes) aportaría datos relevantes acerca de estos comportamientos en otros contextos. Se requieren investigaciones adicionales que exploren estas interacciones.

\section{Nota}

A lo largo de este trabajo, cuando se hace referencia al sexo de las/os participantes se siguen las sugerencias de las normas APA ( $6^{\circ}$ Edición), comprometidas con el aseguramiento de un trato justo a los individuos y los grupos. De esta manera, esas características se especificarán y no se utilizarán categorías generales, con la intención de evitar prejuicios contra las personas en virtud de su sexo, edad y/o grupo socioeconómico.

\section{Referencias}

American Psychological Association (1992). Ethical principles of psychologists and code of conduct. American Psychologist, 57, 1060-1073.

Andrés, M. L., Stelzer, F., Vernucci, S., Juric, L. C., Galli, J. I., \& Guzmán, J. I. N. (2017). Regulación emocional y habilidades académicas: relación en niños de 9 a 11 años de edad. Suma Psicológica, 24(2), 79-86. https://doi.org/10.1016/j.sumpsi.2017.07.001

Bornstein, M. H., Hahn, C. S., Putnick, D. L., \& Pearson, R. (2019). Stability of child temperament: multiple moderation by child and mother characteristics. British Journal of Developmental Psychology, 37(1), 51-67. https://doi.org/10.1111/bjdp.12253

Bridgett, D. J., Oddi, K. B., Laake, L. M., Murdock, K. W., \& Bachmann, M. N. (2013). Integrating and differentiating aspects of self-regulation: effortful control, executive functioning, and links to negative affectivity. Emotion, 13(1), 47-63. https://doi. org/10.1037/a0029536

Conejero, Á., \& Rueda, M. R. (2018). Infant temperament and family socio-economic status in relation to the emergence of attention regulation. Scientific Reports, 8(1), 1-11. https://doi. org/10.1038/s41598-018-28831-x

Cordon, I. M., Melinder, A. M., Goodman, G. S., \& Edelstein, R. S. (2013). Children's and adults' memory for emotional pictures: examining age-related patterns using the Developmental Affective Photo System. Journal of Experimental Child Psychology, 114(2), 339-356. https://doi.org/10.1016/j.jecp.2012.08.004

Derryberry, D., \& Rothbart, M.K. (1997). Reactive and effortful processes in the organization of temperament. Development and Psychopathology, 9(4), 633-652. https://doi.org/10.1017/ S0954579497001375 
Evans, D. E., \& Rothbart, M. K. (2009). A two-factor model of temperament. Personality and Individual Differences, 47(6), 565570. https://doi.org/10.1016/j.paid.2009.05.010

Fernando, J. W., Kashima, Y., \& Laham, S. M. (2015). Alternatives to the fixed-set model: a review of appraisal models of emotion. Cognition and Emotion, 31(1), 19-32. https://doi.org/10.1080/0 2699931.2015.1074548

Fields-Olivieri, M. A., Cole, P. M., \& Maggi, M. C. (2017). Toddler emotional states, temperamental traits, and their interaction: associations with mothers' and fathers' parenting. Journal of Research in Personality, 67, 106-119. https://doi.org/10.1016/j. jrp.2016.05.007

Fivush, R., \& Baker-Ward, L. (2005). The search for meaning: developmental perspectives on internal state language in autobiographical memory. Journal of Cognition and Development, 6(4), 455-462. https://doi.org/10.1207/s15327647jcd0604_1

Gartstein, M. A., Putnam, S. P., \& Rothbart, M. K. (2012). Etiology of preschool behavior problems: contributions of temperament attributes in early childhood. Infant Mental Health Journal, 33(2). 197-211. https://doi.org/10.1002/imhj.21312

Graham, A., Powell, M., Taylor, N., Anderson, D., \& Fitzgerald, R. (2013). Ethical research involving children. Florencia: Unicef Office of Research-Innocenti.

Holodynski, M., \& Seeger, D. (2019). Expressions as signs and their significance for emotional development. Developmental Psychology, 55(9), 1812. https://doi.org/10.1037/dev0000698

Jaume, L. C., Ruetti, E. M., Segretin, M. S., \& Lipina, S. J. (2016). Efectos de la edad sobre la valoración emocional durante la etapa preescolar. Anuario de Investigaciones de la Facultad de Psicología-UBA, 23(2), 281-287.

Kuppens, P., Champagne, D., \& Tuerlinckx, F. (2012). The dynamic interplay between appraisal and core affect in daily life. Frontiers in Psychology, 3, 380. https://doi.org/10.3389/ fpsyg. 2012.00380

Lemerise, E. A., \& Arsenio, W. F. (2000). An integrated model of emotion processes and cognition in social information processing. Child Development, 71(1), 107-118. https://doi.org/10.1111/14678624.00124

Lengua, L. J., \& Long, A. C. (2002). The role of emotionality and self-regulation in the appraisal-coping process: tests of direct and moderating effects. Journal of Applied Developmental Psychology, 23(4), 471-493. https://doi.org/10.1016/S01933973(02)00129-6

Lengua, L. J., Sandler, I. N., West, S. G., \& Wolchik, S. A. (1999). Emotionality and self-regulation, threat appraisal, and coping in children of divorce. Development and Psychopathology, 11(1), 15-37. https://doi.org/10.1017/S0954579499001935

MacNeill, L. A., \& Pérez-Edgar, K. (2020). Temperament and emotion. En S. Hupp, \& J. D. Jewell (Eds.), The Encyclopedia of Child and Adolescent Development (pp. 1-12). Nueva York: John Wiley \& Sons. https://doi.org/10.1002/9781119171492.wecad180

Osa, N. de la, Granero, R., Penelo, E., Domènech, J. M., \& Ezpeleta, L. (2014). The short and very short forms of the Children's Behavior Questionnaire in a community sample of preschoolers. Assessment, 21(4), 463-476. https://doi. org/10.1177/1073191113508809

Parsafar, P., \& Davis, E. L. (2018). Intrapersonal emotion regulation processes influence what children remember about their emotional experiences. Child Development, 90(6), 1935-1951. https://doi.org/10.1111/cdev.13070

Posner, M. I., \& Rothbart, M. K. (2018). Temperament and brain networks of attention. Philosophical Transactions of the Royal Society B: Biological Sciences, 373(1744), 20170254. https://doi. org/10.1098/rstb.2017.0254
Posner, M. I., Rothbart, M. K., Sheese, B. E., \& Voelker, P. (2014). Developing attention: behavioral and brain mechanisms. Advances in Neuroscience, 2014, 1-9. https://doi.org/10.1155/2014/405094

Putnam, S. P., \& Rothbart, M. K. (2006). Development of short and very short forms of the Children's Behavior Questionnaire. Journal of Personality Assessment, 87(1), 102-112. https://doi. org/10.1207/s15327752jpa8701_09

Reyna, C., \& Brussino, S. A. (2015). Diferencias de edad y género en comportamiento social, temperamento y regulación emocional en niños argentinos. Acta Colombiana de Psicología, 18(2), 5164. https://doi.org/10.14718/ACP.2015.18.2.5

Ridderinkhof, K. R. (2017). Emotion in action: a predictive processing perspective and theoretical synthesis. Emotion Review, 9(4), 319-325. https://doi.org/10.1177/1754073916661765

Roseman, I. J., \& Smith, C. A. (2001). Appraisal theory. Appraisal Processes in Emotion: Theory, Methods, Research, 3-19.

Rothbart, M. K. (2011). Becoming who we are: Temperament and personality in development. Guilford Press.

Rothbart, M. K., Ahadi, S. A., Hershey, K. L., \& Fisher, P. (2001). Investigations of temperament at three to seven years: The Children's Behavior Questionnaire. Child Development, 72(5), 1394-1408. https://doi.org/10.1111/1467-8624.00355

Rothbart, M. K., Bates, J. E., Damon, W., \& Lerner, R. (2006). Handbook of child psychology. Social, Emotional, and Personality Development, 6, 99-166.

Rothbart, M. K., Sheese, B. E., \& Posner, M. I. (2007). Executive attention and effortful control: Linking temperament, brain networks, and genes. Child Development Perspectives, 1, 2-7. https://doi.org/10.1111/j.1750-8606.2007.00002.x

Rothbart, M. K., Sheese, B. E., Rueda, M. R., \& Posner, M. I. (2011). Developing mechanisms of self-regulation in early life. Emotion $R e-$ view, 3(2), 207-213. https://doi.org/10.1177/1754073910387943

Rueda, M. R., \& Rothbart, M. K. (2009). The influence of temperament on the development of coping: the role of maturation and experience. New Directions for Child and Adolescent Development, 2009(124), 19-31. https://doi.org/10.1002/cd.240

Ruetti, E., Segretin, M. S., Ramírez, V. A., \& Lipina, S. J. (2019). Role of emotional appraisal in episodic memory in a sample of Argentinean preschoolers. Frontiers in Psychology, 10, 2556. https://doi.org/10.3389/fpsyg.2019.02556

Scherer, K. R., \& Fontaine, J. R. (2018). The semantic structure of emotion words across languages is consistent with componential appraisal models of emotion. Cognition and Emotion, 33(4), 673-682. https://doi.org/10.1080/02699931.2018.1481369

Scherer, K. R., \& Moors, A. (2017). The emotion process: event appraisal and component differentiation. Annual Review of Psychology, 70(1). https://doi.org/10.1146/annurev-psych-122216-011854

Stifter, C., \& Dollar, J. (2016). Temperament and developmental psychopathology. En D. Cicchetti (Ed.), Developmental Psychopathology (pp. 1-62). Hoboken, NJ: John Wiley \& Sons. https:// doi.org/10.1002/9781119125556.devpsy411

Thompson, S. F., Zalewski, M., \& Lengua, L. J. (2014). Appraisal and coping styles account for the effects of temperament on pre-adolescent adjustment. Australian Journal of Psychology, 66(2), 122-129. https://doi.org/10.1111/ajpy.12048

Wang, Q. (2008). Emotion knowledge and autobiographical memory across the preschool years: a cross-cultural longitudinal investigation. Cognition, 108(1), 117-135. https://doi.org/10.1016/j. cognition.2008.02.002

Yih, J., Uusberg, A., Taxer, J. L., \& Gross, J. J. (2019). Better together: a unified perspective on appraisal and emotion regulation. Cognition and Emotion, 33(1), 41-47. https://doi.org/10.10 80/02699931.2018.1504749 